\title{
Isolated congenital auditory ossicle malformation
}

INSERM

\section{Source}

INSERM. (1999). Orphanet: an online rare disease and orphan drug data base. Isolated congenital auditory ossicle malformation. ORPHA:162526

Isolated congenital auditory ossicle malformation is a rare, congenital, middle ear anomaly characterized by, usually unilateral and sporadic, variations in the number, size and/or configuration of the ossicles, with no tympanic membrane and external ear abnormalities and no history of trauma or infection. Patients frequently present late, after schooling has started, with non-progressive, conductive hearing loss often associated with speech delay and poor school performance. 